\title{
Quaderni
}

QUADERNI Communication, technologies, pouvoir

77 | Hiver 201 1-2012

L'évaluation de la recherche

\section{L'impact stratégique de la consommation online des hôpitaux : Le cas du cancer dans les pays européens francophones}

Pablo Medina

\section{(2) OpenEdition \\ Journals \\ Édition électronique \\ URL : http://journals.openedition.org/quaderni/571 \\ DOI : 10.4000/quaderni.571 \\ ISSN : 2105-2956 \\ Éditeur \\ Les éditions de la Maison des sciences de l'Homme}

\section{Édition imprimée}

Date de publication : 5 janvier 2012

Pagination : 121-130

Référence électronique

Pablo Medina, «L'impact stratégique de la consommation online des hôpitaux : Le cas du cancer dans les pays européens francophones », Quaderni [En ligne], 77 | Hiver 2011-2012, mis en ligne le 05 janvier 2014, consulté le 21 avril 2019. URL : http://journals.openedition.org/quaderni/571 ; DOI : 10.4000/ quaderni.571 


\section{Communication}

\section{l'impact stratégique de}

la consommation online des hôpitaux Le cas du cancer dans les pays européens francophones

\section{Pablo Medina}

Faculté de Sciences Économiques Université de Neuchâtel, Suisse
L'Internet est devenu une source d'information médicale de plus en plus utilisée par les patients, d'où l'importance que les hôpitaux établissent une vraie stratégie de communication online. Le but de cet article est de comprendre comment les hôpitaux des pays francophones en Europe adaptent leurs stratégies de communication online aux besoins communicationnels et médicaux des patients oncologiques. Pour ce faire, on réalise, en premier lieu, une révision bibliographique sur les stratégies de communication online mises en place par les hôpitaux; et, en deuxième lieu, on s'attarde sur une étude de cas portant sur la communication online sur le cancer du sein développée par 55 hôpitaux francophones de France, Belgique, Suisse et Luxembourg.

\section{La communication online des hôpitaux}

L'accès libre aux informations médicales partagées sur Internet est un défi, et l'absence de formation et de connaissances chez plusieurs utilisateurs est un fait évident ${ }^{1}$. Néanmoins, l'Internet peut contribuer à réduire les inégalités sociales en ce qui concerne la prévention primaire, la promotion de la santé et l'éducation à la santé2. Dans ce contexte, les hôpitaux réfléchissent aux stratégies de communication online leur permettant d'optimiser l'utilisation d'Internet, notamment, l'utilisation de la page Web comme un outil de communication à part entière. Ces stratégies se basent sur cinq concepts différents et complémentaires : caractère pédagogique, accessibilité, personnalisation, interactivité, et communication interpersonnelle.

En premier lieu, les hôpitaux ont recours à l'approche pédagogique. Le pouvoir d'Internet 
comme outil pédagogique ${ }^{3}$ a mené plusieurs institutions sanitaires à utiliser ce média pour apprendre aux citoyens certaines informations sur les habitudes saines, notamment celles qui concernent les allergies, l'obésité et le cancer4. En deuxième lieu, les hôpitaux considèrent l'accessibilité de la page Web comme un élément clé. Les pages Web de santé considérées comme effectives transmettent une image de qualité fiable, de confiance et de simplicité en ce qui concerne leur utilisation ${ }^{5}$. D'ailleurs, dans ce cadre, les sites Internet qui suscitent des préférences positives grâce à leur design visuel génèrent aussi des jugements positifs en ce qui concerne leur crédibilité ${ }^{6}$. Donc, l'accessibilité et le design déterminent la stratégie de communication online de l'hôpital, laquelle est aussi influencée par le concept de la précision informative. Autrement dit, les sites Internet transmettant des informations sanitaires doivent évoluer de la diffusion d'informations générales à la satisfaction des besoins informatifs précis des usagers ${ }^{7}$. En troisième lieu, les hôpitaux diffusent des informations personnalisées. La demande d'informations médicales personnalisées est une réalité de plus en plus importante ${ }^{8}$, notamment parce que l'usage de l'Internet pour se procurer des informations sanitaires est très lié à l'intimité des expériences de santé vécues par les patients 9 .

En quatrième lieu, les pages Web des hôpitaux deviennent de plus en plus interactives. Sur Internet, le patient priorise la recherche d'expériences sur la recherche d'informations ${ }^{10}$, ce qui a mené plusieurs hôpitaux à développer sur leur page Web des applications 2.0 (forums, blogs, présence sur des médias sociaux tels que Facebook, etc.). Ces applications, d'une part, permettent au citoyen de garder et de partager les informations médicales le concernant ${ }^{11}$ et, d'une autre part, permettent aux institutions sanitaires de mettre en place des campagnes d'éducation sanitaire, notamment celles qui s'adressent aux groupes défavorisés (personnes handicapées), aux personnes âgées, ou aux personnes habitant dans des endroits isolés d'un point de vue géographique $^{12}$. Et finalement, en cinquième lieu, la stratégie de communication online de l'hôpital se base sur la possibilité offerte au patient de parler avec le professionnel de la santé sur place, à l'hôpital (communication interpersonnelle). L'Internet ne peut être isolé des autres sources d'information de santé utilisées au quotidien par le patient, comme par exemple les médecins et les médias traditionnels ${ }^{13}$, d'où l'importance que la page Web de l'hôpital propose aux patients différents moyens de contacter le professionnel de la santé (numéro de téléphone, email, prise de rendez-vous sur place, etc.).

\section{La communication hospitalière online sur le cancer : méthodologie}

L'objectif de cet article est, d'une part, d'évaluer d'un point de vue communicatif et médical la page Web des hôpitaux francophones en Europe traitant le cancer du sein et, d'une autre part, d'établir des consignes permettant à ces hôpitaux de mettre à jour des pages Web qui aident davantage le patient à mieux décider de l'institution hospitalière où se faire traiter les pathologies concernant le cancer du sein. Pour ce faire, on a choisi 55 hôpitaux, dont 35 en France, 9 en Belgique, 7 en Suisse et 4 au Luxembourg ${ }^{14}$. Les critères utilisés pour sélectionner les hôpitaux se sont basés sur le nombre de lits et sur le statut 
public ou privé de chaque hôpital. Ces critères se sont appliqués d'une manière différente dans chacun des quatre pays, compte tenu des différences existantes concernant la population et le régime sanitaire du pays. En 2007, la population en France était de 63392100 habitants, de 10584500 habitants en Belgique, de 7508700 habitants en Suisse et de 476200 habitants au Luxembourg $^{15}$. En ce qui concerne la France, on a choisi hôpitaux publics ayant plus de 1000 lits, ce qui est justifié par le fait que le système sanitaire français se base principalement sur les hôpitaux publics, et qu'en choisissant les hôpitaux à plus de 1000 lits on étudie les institutions hospitalières les plus importantes du pays. Par rapport à la Belgique, dont le système de santé se base aussi sur les hôpitaux publics, on a choisi les hôpitaux publics ayant plus de 700 lits. En Suisse, en plus des hôpitaux publics ayant plus de 1000 lits, on a pris aussi les cinq cliniques privées les plus grandes selon leur nombre de lits parce que le système sanitaire suisse se base fortement sur les organisations sanitaires privées. Finalement, en ce qui concerne le Luxembourg, on a sélectionné les hôpitaux publics à plus de 300 lits.

L'analyse de chaque page Web a pour objectif d'évaluer deux aspects : a) l'architecture communicative, et b) le niveau de suivi des recommandations établies par Europa Donna concernant les unités hospitalières spécialisées dans le traitement du cancer du $\operatorname{sein}^{16}$. Afin d'analyser le premier aspect, on a évalué la homepage de chaque hôpital en utilisant 8 critères différents (voir dans la rubrique suivante Tableau 1. Stratégie de communication sur la homepage). L'analyse de la homepage constitue une méthodologie qui a déjà été utilisée par d'autres auteurs pour étudier plusieurs aspects communicationnels comme, par exemple, le contenu scientifique de la homepage ${ }^{17}$; l'orientation de la homepage vers l'information, le client et l'administration ${ }^{18}$; la navigabilité et la disponibilité ${ }^{19}$; l'accessibilité des personnes ayant des handicaps ${ }^{20 ;}$ ou encore le genre de navigation réalisée par les utilisateurs ${ }^{21}$. En ce qui concerne le deuxième aspect d'analyse dans cette étude de cas (recommandations d'Europa Donna), on a analysé les pages Web regroupant toute l'information concernant le traitement du cancer du sein. Pour ce faire, on a utilisé 9 critères (voir dans la rubrique suivante Tableau 2. Page sur le traitement du cancer $d u$ sein. Critères Europa Donna). Finalement, il faut dire que cet article analyse uniquement les informations transmises par chaque hôpital à travers son site Internet.

\section{La communication hospitalière online sur le cancer : résultats}

Les résultats obtenus permettent de mieux comprendre le rôle de la homepage dans les actions de communication online des hôpitaux européens francophones traitant le cancer du sein, ainsi que de mieux connaître le niveau de suivi des recommandations établies par Europa Donna concernant les unités hospitalières spécialisées dans le traitement du cancer du sein. Étant donné les différences existantes entre le nombre d'hôpitaux analysés dans chaque pays, on priorise dans cette rubrique de l'article les pourcentages globaux concernant les 55 hôpitaux étudiés dans les quatre pays.

Par rapport à la homepage, la plupart des hôpitaux analysés parient sur des stratégies favorisant la 
navigabilité (voir plus bas Tableau 1. Stratégie de communication sur la homepage). Ainsi, 65\% des hôpitaux dispose sur la homepage d'un accès patient permettant de diriger celui-ci vers les informations le concernant uniquement ; 36,4\% dispose de menus déroulants permettant au patient de consulter les informations d'une manière plus rapide et plus pratique ; $30,9 \%$ consacre plus d'importance à l'image qu'au texte, ce qui est cohérent avec la société « visuellement intensive » dans laquelle on vit $^{22}$ et ce qui, d'ailleurs, donne lieu à la promotion de la représentation visuelle de la science ${ }^{23}$ et $27,3 \%$ offre au patient la possibilité de consulter la page Web dans plusieurs langues représentatives de la région où se trouve l'hôpital. Par contre, peu d'hôpitaux disposent sur la homepage de liens vers le département d'oncologie $(14,5 \%)$, ou encore vers la page regroupant toutes les informations concernant le traitement du cancer du sein $(5,5 \%)$.

Selon les résultats de cette étude, le nombre d'hôpitaux disposant d'une page Web consacrée au traitement du cancer du sein est de 15 (27,3\% des hôpitaux analysés), dont 7 en France (donc $20 \%$ des hôpitaux français analysés), 5 en Belgique (55,6\% des hôpitaux belges analysés), 3 en Suisse (42,9\% des hôpitaux suisses analysés), et aucun au Luxembourg. Cette analyse priorise les résultats globaux sur l'ensemble des 55 hôpitaux étudiés, c'est pourquoi les pourcentages rapportés plus bas sur les critères d'Europa Donna (voir Tableau 2. Page sur le traitement du cancer $d u$ sein. Critères Europa Donna) ne prennent pas comme référence les 15 hôpitaux disposant d'une page Web pour le traitement du cancer du sein, mais les 55 hôpitaux analysés dans cette étude. De cette manière, on peut apprécier plus clairement le suivi des recommandations d'Europa Donna dans l'ensemble de chacun des 4 pays.

Les recommandations établies par Europa Donna constituent un avancement très important dans le traitement du cancer du sein, d'où l'importance que les hôpitaux disposant d'une unité médicale spécialisée dans le traitement de cette pathologie diffusent sur leur page Web des informations permettant au patient d'évaluer le suivi de ces critères par l'hôpital. Néanmoins, cette étude démontre que les hôpitaux francophones en Europe ne communiquent pas assez sur les recommandations les plus importantes d'Europa Donna, comme par exemple l'identification du responsable de l'unité médicale (14,5\% des hôpitaux) ; l'existence, au moins, de deux radiologues $(9,1 \%)$; l'existence, au moins, de deux membres de l'unité capables d'offrir un appui psychologique au patient $(5,5 \%)$; ou le traitement, d'au moins, 150 nouveaux cas par an (3,6\%). L'accès à la page Web constitue le premier pas d'un processus de consultation d'informations médicales, lequel processus souvent conclut avec l'arrivée du patient oncologique au centre hospitalier, d'où l'importance que la page Web de l'hôpital facilite les rapports communicationnels du patient avec l'institution hospitalière. Et pour ce faire, l'organisation hospitalière peut utiliser plusieurs moyens, comme par exemple l'existence sur la page Web d'un email de contact nominatif ou d'un numéro de téléphone permettant au patient

Tableau 1. Stratégie de communication sur la homepage 


\begin{tabular}{|c|c|c|c|c|c|}
\hline CRITÈRES & $\begin{array}{l}\text { FRANCE } \\
\text { Pourcentages } \\
\text { sur les } 35 \text { hô- } \\
\text { pitaux français } \\
\text { analysés }\end{array}$ & $\begin{array}{l}\text { BELGIQUE } \\
\text { Pourcentages } \\
\text { sur les } 9 \text { hô- } \\
\text { pitaux belges } \\
\text { analysés }\end{array}$ & $\begin{array}{l}\text { SUISSE } \\
\text { Pourcen- } \\
\text { tages sur } \\
\text { les } 7 \text { hôpi- } \\
\text { taux suisses } \\
\text { analysés }\end{array}$ & $\begin{array}{l}\text { LUXEMBOURG } \\
\text { Pourcentages sur } \\
\text { les } 4 \text { hôpitaux } \\
\text { luxembourgeois } \\
\text { analysés }\end{array}$ & $\begin{array}{l}\text { TOTAL } \\
\text { Pourcentages } \\
\text { sur les } 55 \text { hô- } \\
\text { pitaux des } 4 \\
\text { pays }\end{array}$ \\
\hline $\begin{array}{l}\text { 1. Présence d'un } \\
\text { accès patient }\end{array}$ & $68,6 \%$ & $44,4 \%$ & $85,7 \%$ & $50 \%$ & $65,5 \%$ \\
\hline $\begin{array}{l}\text { 2. Existence de me- } \\
\text { nus déroulants }\end{array}$ & $37,1 \%$ & $44,4 \%$ & $28,6 \%$ & $25 \%$ & $36,4 \%$ \\
\hline $\begin{array}{l}\text { 3. L'image a plus } \\
\text { d'espace que le } \\
\text { texte }\end{array}$ & $25,7 \%$ & $44,4 \%$ & $42,9 \%$ & $25 \%$ & $30,9 \%$ \\
\hline $\begin{array}{l}\text { 4. Existence d'une } \\
\text { version de la home- } \\
\text { page dans une lan- } \\
\text { gue autre que le } \\
\text { Français }\end{array}$ & $11,4 \%$ & $55,6 \%$ & $71,4 \%$ & $25 \%$ & $27,3 \%$ \\
\hline $\begin{array}{l}\text { 5. Existence d'une } \\
\text { page regroupant } \\
\text { toutes les informa- } \\
\text { tions sur le cancer } \\
\text { du sein }\end{array}$ & $20 \%$ & $55,6 \%$ & $42,9 \%$ & $0 \%$ & $27,3 \%$ \\
\hline $\begin{array}{l}\text { 6. Lien vers le dé- } \\
\text { partement d'onco- } \\
\text { logie }\end{array}$ & $11,4 \%$ & $22,2 \%$ & $14,3 \%$ & $25 \%$ & $14,5 \%$ \\
\hline $\begin{array}{l}\text { 7. Lien vers une } \\
\text { page sur le traite- } \\
\text { ment du cancer du } \\
\text { sein }\end{array}$ & $5,7 \%$ & $11,11 \%$ & $0 \%$ & $0 \%$ & $5,5 \%$ \\
\hline $\begin{array}{l}\text { 8. Existence de } \\
\text { logos signalant la } \\
\text { présence de l'hôpi- } \\
\text { tal dans les réseaux } \\
\text { sociaux (Facebook, } \\
\text { Twitter, etc.) }\end{array}$ & $0 \%$ & $0 \%$ & $0 \%$ & $14,3 \%$ & $1,8 \%$ \\
\hline
\end{tabular}




\begin{tabular}{|c|c|c|c|c|c|}
\hline CRITÈRES & $\begin{array}{l}\text { FRANCE } \\
\text { Pourcentages } \\
\text { sur les } 35 \text { hô- } \\
\text { pitaux fran- } \\
\text { çais analysés }\end{array}$ & $\begin{array}{l}\text { BELGIQUE } \\
\text { Pourcentages } \\
\text { sur les } 9 \text { hô- } \\
\text { pitaux belges } \\
\text { analysés }\end{array}$ & $\begin{array}{l}\text { SUISSE } \\
\text { P o u r e n - } \\
\text { tages sur les } \\
7 \text { hôpitaux } \\
\text { suisses ana- } \\
\text { lysés }\end{array}$ & $\begin{array}{l}\text { LUXEMBOURG } \\
\text { Pourcentages sur les } \\
4 \text { hôpitaux luxem- } \\
\text { bourgeois analysés }\end{array}$ & $\begin{array}{l}\text { TOTAL } \\
\text { Pourcen- } \\
\text { tages sur } \\
\text { les } 55 \text { hô- } \\
\text { pitaux des } \\
4 \text { pays }\end{array}$ \\
\hline $\begin{array}{l}\text { 1. Existence d'un em- } \\
\text { ail de contact nomina- } \\
\text { tif ou d'un numéro de } \\
\text { téléphone }\end{array}$ & $17,1 \%$ & $55,6 \%$ & $28,6 \%$ & $0 \%$ & $23,6 \%$ \\
\hline $\begin{array}{l}\text { 2. Identification du res- } \\
\text { ponsable de l'unité }\end{array}$ & $5,7 \%$ & $44,4 \%$ & $28,6 \%$ & $0 \%$ & $14,5 \%$ \\
\hline $\begin{array}{l}\text { 3. L'unité dispose d'au } \\
\text { moins deux radiolo- } \\
\text { gues }\end{array}$ & $2,9 \%$ & $33,3 \%$ & $14,3 \%$ & $0 \%$ & $9,1 \%$ \\
\hline $\begin{array}{l}\text { 4. L'unité dispose d'au } \\
\text { moins deux membres } \\
\text { capables d'offrir un } \\
\text { appui psychologique } \\
\text { au patient }\end{array}$ & $0 \%$ & $11,1 \%$ & $28,6 \%$ & $0 \%$ & $5,5 \%$ \\
\hline $\begin{array}{l}\text { 5. Existence d'une date } \\
\text { de mise à jour des in- } \\
\text { formations }\end{array}$ & $5,7 \%$ & $0 \%$ & $14,3 \%$ & $0 \%$ & $5,5 \%$ \\
\hline $\begin{array}{l}\text { 6. L'unité traite au } \\
\text { moins } 150 \text { nouveaux } \\
\text { cas par an }\end{array}$ & $0 \%$ & $11,1 \%$ & $14,3 \%$ & $0 \%$ & $3,6 \%$ \\
\hline $\begin{array}{l}\text { 7. Le patient peut } \\
\text { télécharger sur la } \\
\text { page Web un rapport } \\
\text { concernant l'activité } \\
\text { de l'unité }\end{array}$ & $2,9 \%$ & $0 \%$ & $0 \%$ & $0 \%$ & $1,8 \%$ \\
\hline $\begin{array}{l}\text { 8. L'unité dispose } \\
\text { d'une équipe de soins } \\
\text { de support }\end{array}$ & $0 \%$ & $0 \%$ & $0 \%$ & $0 \%$ & $\mathbf{0} \%$ \\
\hline $\begin{array}{l}\text { 9. L'unité assure des } \\
\text { consultations dans des } \\
\text { établissements plus } \\
\text { petits }\end{array}$ & $0 \%$ & $0 \%$ & $0 \%$ & $0 \%$ & $0 \%$ \\
\hline
\end{tabular}


Tableau 2. Page sur le traitement du cancer du sein. Critères Europa Donna*

*Tous les critères se basent sur les recommandations établies par Europa Donna, lesquelles sont disponibles sur la page Web officielle de l'organisation (www.europadonna.fr). Les deux seuls critères qui ne se basent pas sur les recommandations de cet organisme et qui ont donc été ajoutés par l'auteur sont le critère 1 (existence d'un email de contact) et le critère 5 (date de mise à jour des informations).

de communiquer avec le centre hospitalier d'une manière plus personnelle. Pourtant, cette réalité n'est pas trop respectée par les hôpitaux analysés puisque uniquement le 23,6\% des hôpitaux étudiés dispose de l'un de ces deux outils de contact dans la page Web consacrée au traitement du cancer du sein.

\section{Conclusion}

La révision bibliographique réalisée sur le domaine de la communication online des hôpitaux, et l'étude de cas menée sur la page Web de 55 hôpitaux européens francophones nous permettent de signaler trois dernières idées concernant ce sujet. En premier lieu, la structure de la homepage des hôpitaux n'est pas assez orientée vers le patient, le besoin de diffuser des informations institutionnelles sur l'hôpital l'emporte sur le besoin du patient de recevoir des informations précises. Autrement dit, les hôpitaux doivent mieux prendre en considération l'approche pédagogique ainsi que les concepts d'accessibilité et de personnalisation de l'information. En deuxième lieu, les pages regroupant toutes les informations sur le traitement du cancer du sein ont besoin d'adopter une approche plus quantitative qui se traduise par la diffusion de chiffres d'activité de l'unité hospitalière permettant au patient de mieux comprendre la performance médicale de ce département. De cette manière, le niveau de crédibilité de l'hôpital augmente grâce à la diffusion d'informations objectives mettant en avant le savoir-faire médical de l'unité ; ce qui permet, d'une part, de mieux satisfaire les besoins informationnels du patient et, d'une autre part, d'informer d'une manière plus efficace sur l'engagement de l'hôpital avec le respect des recommandations établies par Europa Donna. Et en troisième lieu, il faut mettre en avant l'importance que les hôpitaux parient davantage sur l'interactivité de la page Web ainsi que sur les outils permettant au patient de communiquer avec le médecin d'une manière personnelle (communication interpersonnelle). 
$\mathrm{N} \cdot \mathrm{O} \cdot \mathrm{T} \cdot \mathrm{E} \cdot \mathrm{S}$

1. Keiko Kishimoto, Noriko Fukushima, «Use of anonymous web communities and websites by medical consumers in Japan to research drug information ", in Yakugaku Zasshi, 2011, vol. 131, n 5, pp. 685-95. 2. Émilie Renahy, Emmanuelle Cadot, Christelle Roustit, Isabelle Parizot, Pierre Chauvin, «Recherche d'information en santé sur l'Internet : une analyse contextuelle des données SIRS, une cohorte parisienne », in Santé Publique, 2009, vol. 21, hs2, pp. 27-40.

3. Francisco Sierra Caballero, Comunicación, educación y desarrollo : apuntes para una historia de la comunicación educativa, Sevilla, Comunicación Social, 2002.

4. María Natividad Moyá Garrido, Internet, salud y ciudadanía, Sevilla, Agencia de Evaluación de Tecnologías Sanitarias, 2009.

5. Julie Fisher, Frada Burstein, Kathy Lynch, Kate Lazarenko, «Usability + usefulness = trust : an exploratory study of Australian health web sites $»$, in Internet Research, 2008, vol. 18, n 5, pp. 477-498.

6. David Robins, Jason Holmes, Mary Stansbury, "Consumer health information on the web the relationship of visual design and perceptions of credibility », in Journal of the American Society for Information Science and Technology, 2010, vol. 61, $\mathrm{n}^{\mathrm{o}}$ 1, pp. 13-29.

7. Paul Huntington, David Nicholas, Peter Williams, «Characterising and profiling health web user and site types : going beyond hits », in Aslib Proceedings, 2003, vol. 55, $\mathrm{n}^{\mathrm{o}}$ 5/6, pp. 277-289.

8. Dean Giustini, «How Web 2.0 is changing medicine ", in British Medical Journal, 2006, vol. 333, $\mathrm{n}^{\circ}$ 7582, pp. 1283-1284.

9. Joëlle Kivits, «Everyday health and the Internet : a mediated health perspective on health information seeking », in Sociology of Health \& Illness, 2009, vol. 31, no 5, pp. 673-687.

10. Patrick Buckley, The complete guide to hospital marketing, Marblehead, HCPro Inc, 2007.

11. Gary Bennett, Russell Glasgow, «The delivery of public health interventions via the Internet : actualizing their potential », in Annual Review of Public Health, 2009, vol. 30, pp. 273-292.

12. Maged Kamel Boulos, Steve Wheeler, «The emerging Web 2.0 social software : an enabling suite of sociable technologies in health and health care education », in Health Information and Libraries Journal, 2007, vol. 24, no 1 , pp. 2-23.

13. Marc Lemire, «Internet et responsabilisation : perspective de l'usager au quotidien ", in Santé Publique, 2009, vol. 21, hs2, pp.13-25.

14. Les hôpitaux français analysés ont été : 1) Assistance Publique - Hôpitaux de Paris, 2) Hôpitaux de Lyon, 3) Assistance Publique - Hôpitaux de Marseille, 4) CHU de Bordeaux, 5) CHU de Nantes, 6) CHRU de Lille, 7) CHU de Toulouse, 8) CHU de Brest, 9) CHU de Rouen, 10) Hôpitaux Universitaires de Strasbourg, 11) CHRU de Montpellier, 12) CHRU de Tours, 13) CHU de Grenoble, 14) CHU de Limoges, 15) CHR de Metz-Thionville, 16) CHU de Clermont-Ferrand, 17) CHU de Rennes, 18) CHU de Saint-Étienne, 19) CHU de Nîmes, 20) CHU de Dijon, 21) CHU de Nice, 22) CHU de Nancy, 23) CH du Mans, 24) CHU de Poitiers, 25) CHU d'Amiens, 26) CHU de Caen, 27) CHU d'Angers, 28) CH de Mulhouse, 29) CHR d'Orléans, 30) CH de La Rochelle, 31) CHU de Besançon, 32) CH de la Côte Basque, 33) Centre Hospitalier Intercommunal de Toulon, 34) CH de Perpignan et 35) CH de Béziers. En Belgique, on a analysé : 1) CHU de Charleroi, 2) Grand Hôpital de Charleroi, 3) Centre Hospitalier Chrétien, 4) Cliniques Universitaires Saint-Luc, 5) CHU de Liège, 6) Hôpital Erasme, 7) CHU Brugmann, 8) Hôpitaux Iris Sud et 9) UZ Brussel. En Suisse, les hôpitaux évalués 
ont été : 1) Hôpitaux Universitaires de Genève, 2) Centre Hospitalier Universitaire Vaudois, 3) Clinique de la Source (Swish Leading Hospitals), 4) Clinique de Genolier, 5) Clinique Générale Beaulieu (Swish Leading Hospitals), 6) Clinique Cecil (Groupe de cliniques privées Hirslanden) et 7) Clinique Bois-Cerf (Groupe de cliniques privées Hirslanden). Finalement, au Luxembourg on a analysé : 1) Centre Hospitalier Emile Mayrisch, 2) Centre Hospitalier du Luxembourg, 3) Centre Hospitalier du Nord et 4) Centre Hospitalier du Kirchberg. Dans cette liste par pays, les hôpitaux sont classés selon leur nombre de lits, à commencer par l'hôpital le plus grand. Cette analyse a été réalisée entre le 9 et le 30 juin 2010.

15. Giampolo Lanzieri, Population in Europe 2007 : first results. Eurostat. Statistics in focus 81/2008, population and social conditions, Luxembourg, Eurostat, 2008.

16. Europa Donna est une coalition européenne qui lutte contre le cancer du sein. Elle a été créée en 1993 et rassemble aujourd'hui 46 pays. Cet organisme a établi une série de recommandations pour les unités hospitalières spécialisées dans le traitement du cancer du sein. La liste complète de ces recommandations peut être consultée à la page officielle de cet organisme : http://www.europadonna.fr. Page consultée le 9 septembre 2011.

17. Kitty Dumont, Wolfgang Frindte, «Content analysis of the homepages of academic psychologist », in Computers in Human Behavior, 2005, vol. 21, $\mathrm{n}^{\mathrm{o}} 1$, pp. 73-83.

18. Chi Chang, « The e-hospital website measure architecture approach : integrating internal and external customers' needs in information delivery services ", in Asian Journal of Health and Information Sciences, 2007, vol. 2, no 1-4, pp. 116-131.

19. J. Seitz, M. Siegmund, M. Völk, S. Feuerbach, M. Strotzer, « Evaluation of the internet presence of diagnostic radiology units at German universities " in Der Radiologe, 2002, vol. 42, n 9, pp. 739-744.

20. Stephanie Hackett, Bambang Parmanto, «A longitudinal evaluation of accessibility : higher education web sites », in Internet Research, 2005, vol. 15, n 3, pp. 281-294.

21. Mohammad Rezailashkajani, Delnaz Roshandel, Shahin Ansari, Mohammad Zali, «A web-based patient education system and self-help group in Persian language for inflammatory bowel disease patients ", in International Journal of Medical Informatics, 2008, vol. $77, \mathrm{n}^{\circ}$ 2, pp. 122-128.

22. Paul Martin Lester, Visual communication : images with messages, Belmont, Wadsworth Pub. Co, 1995. 23. Luc Pauwels (Ed.), Visual cultures of science : rethinking representational practices in knowledge building and science communication, Hanover, Dartmouth College Press, 2005. 


\section{$R \cdot E ́ \cdot S \cdot U \cdot M \cdot E$}

Le rôle d'Internet comme source d'information médicale joue un rôle essentiel pour les citoyens, mais aussi pour les hôpitaux. Ces institutions parient sur le développement d'une page Web comme stratégie pour améliorer leurs rapports avec les patients, notamment avec les patients oncologiques. Dans cet article, on réalise une révision bibliographique sur la communication online des hôpitaux ainsi qu'une étude de cas sur le rôle de la page Web des hôpitaux européens francophones (France, Belgique, Suisse et Luxembourg) dans leurs rapports avec les patients souffrant d'un cancer. La conclusion de l'étude est que les hôpitaux doivent faire davantage d'efforts pour mettre en place des pages Web vraiment orientées vers le patient, et réellement basées sur la divulgation de chiffres ainsi que sur la hiérarchisation des informations.

\footnotetext{
Abstract

The role of the Internet as a source of medical information is essential for citizens, but also for hospitals. These institutions are betting on the development of a web page as a strategy enabling them to improve their relationships with patients, especially patients with cancer. In this paper, we realize a bibliographic review on the online communication of hospitals and a case study on the role of the web page European hospitals French (France, Belgium, Switzerland and Luxembourg) in their relationships with patients suffering from a cancer. The conclusion of the study is that hospitals must make greater efforts to develop Web pages oriented really patient and really based on the release of figures as well as the hierarchy of information.
} 